\title{
The Urban Health Equity Assessment and Response Tool (HEART) - a Decade of Development and Implementation
}

\author{
Amit Prasad
}

Published online: 27 September 2018

(C) The New York Academy of Medicine 2018

As a majority of the world's population lives in urban areas since 2008, cities are increasingly at the forefront of tackling critical global development issues, including public health. Making cities inclusive, safe, sustainable, and resilient is one of the 17 Sustainable Development Goals (SDGs) adopted by Member States of the United Nations in 2015. Other SDG keys to achieving this goal are important determinants of health such as housing, disaster management, and access to green spaces. There are a number of common goals between the SDG relating to cities and the SDG on ensuring healthy lives and well-being including road safety, air pollution, and equity.

The percentage of the world's population living in urban areas is projected to increase from 54\% in 2015 to $60 \%$ in 2030 and to $66 \%$ by 2050 . Sub-Saharan Africa (SSA) will be the fastest urbanizing region between 2015 and 2050. Urban population as a proportion of total population will increase in SSA from $37.9 \%$ in 2015 to $54.8 \%$ in 2050.

In its 2008 World Health Report, the World Health Organization (WHO) identified the effects of illmanaged urbanization as one of the three biggest challenges for public health in the twenty-first century. The second joint WHO-UN Habitat report on urban health (2016) emphasized the role of effective urban governance to tackle challenges related to health and health

\section{A. Prasad $(\bowtie)$}

Indian Institute of Technology Mandi, Parashar Road, Tehsil Sadar, Near Kataula, Kamand, Himachal Pradesh 175005, India e-mail: prasada@who.int equity. Effective governance can only ensue from a combined effort of a multitude of actors including different levels of government, NGOs, the private sector, and the community.

In this special edition focused on the Urban Health Equity Assessment and Response Tool (Urban HEART), we start with an overview of the importance of health and health equity in the era of the SDGs. The first paper in the series outlines the case for using evidence-based action across multiple sectors to tackle health inequalities in cities. A systematic review of tools on the physical environment relating to urban health is provided in the second paper. Urban HEART was developed jointly by WHO, city and national officials, and academics and researchers in 2010 to support stakeholders in cities address health inequities by acting on the social, economic, and environmental determinants of health. More than 100 cities in 53 countries had used the tool to plan action on health inequities by the end of 2015 .

The remaining four papers demonstrate the scope of actions undertaken to reduce health inequities using Urban HEART in different political, economic, social, and geographically diverse contexts. They are focused on the experiences of Barcelona, Detroit, Matsapha, and Tehran with each city facing common as well as unique challenges relating to their context. While the papers express a wide range of urban health challenges, they also prove that technical solutions for many of the challenges exist. To be successful, however, different sectors, including those within and outside of health, would need to work together. These four cities demonstrate both what is feasible and the successes that are possible. 